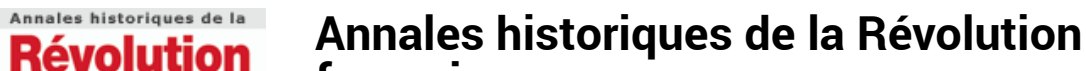

française française

352 | avril-juin 2008

Les temps composés de l'économie

\section{Les transports intérieurs sous la Révolution : une politique de l'espace}

Domestic Transport under the Revolution: the Politics of Space

\section{Anne Conchon}

\section{(2) OpenEdition \\ 1 Journals}

\section{Édition électronique}

URL : https://journals.openedition.org/ahrf/11061

DOI : 10.4000/ahrf.11061

ISSN : 1952-403X

Éditeur :

Armand Colin, Société des études robespierristes

\section{Édition imprimée}

Date de publication : 1 juin 2008

Pagination : 5-28

ISBN : 978-2200-92514-7

ISSN : 0003-4436

Référence électronique

Anne Conchon, «Les transports intérieurs sous la Révolution : une politique de l'espace ", Annales historiques de la Révolution française [En ligne], 352 | avril-juin 2008, mis en ligne le 01 juin 2011, consulté le 22 avril 2022. URL : http://journals.openedition.org/ahrf/11061 ; DOI : https://doi.org/ 10.4000/ahrf.11061 


\title{
LES TRANSPORTS INTÉRIEURS SOUS LA RÉVOLUTION : UNE POLITIQUE DE L'ESPACE
}

Anne CONCHON

\begin{abstract}
Si la Révolution s'inscrit assurément par de nombreux aspects dans la continuité de la politique des transports engagée par la monarchie au cours du XVIII ${ }^{e}$ siècle, la période est marquée par un ralentissement notable des constructions et par la détérioration des infrastructures existantes. Les autorités révolutionnaires ont cependant entrepris de promouvoir le projet d'un espace homogène de circulation qui serait sous-tendu par un réseau de communications, étalonné par la seule distance et entretenu grâce à la péréquation des recettes péagères, dans le but de contribuer à l'unité du territoire.
\end{abstract}

Mots-clés : Révolution française, transports, espace, routes, canaux, navigation fluviale.

Tout au long du XVIII ${ }^{e}$ siècle, la monarchie avait mené une active politique de transports sous la direction des Ponts et Chaussées, avec un vaste programme de constructions routières et l'aménagement de nombreuses voies d'eau. Parallèlement, toute une série de dispositions réglementaires fondaient une législation routière (hiérarchie des axes selon leur intérêt économique, codification de la largeur des routes, plantation d'arbres...). La Révolution s'inscrit assurément dans la continuité de cette politique de promotion de communications sûres et rapides, autour desquelles gravite un nombre croissant d'entreprises et d'activités. La pression conjuguée des difficultés frumentaires et des impératifs militaires va cependant faire des transports un enjeu stratégique. La guerre, qui freina les relations avec plusieurs pays voisins et entraîna le blocus des ports, amena l'État à se recentrer sur les liaisons intérieures. 
La question des transports suscita un nombre assez important de travaux. Parmi eux, le premier fascicule de l'Atlas de la Révolution française, consacré aux routes et communications, avait clairement considéré la Révolution française sur la longue durée dans le sillage de la politique engagée par la monarchie au XVIII ${ }^{\mathrm{e}}$ siècle $^{1}$. Dans cette perspective est-il pertinent de traiter la Révolution comme un moment à part entière en ce qui concerne l'économie des transports ? Les jalons de l'intense activité législative et réglementaire qui caractérise la période sont bien connus dans l'ensemble. L'organisation administrative des Ponts et Chaussées et le corps des ingénieurs ont suscité plusieurs études ${ }^{2}$. Les inflexions majeures en matière de financement des infrastructures - les ressources de l'impôt puis la tarification à l'usage par le biais du péage - sont également bien étudiées. C'est sans doute la question de la création de la taxe d'entretien et de la mise en place des barrières qui a fait l'objet des recherches les plus nombreuses ${ }^{3}$. Le montant des recettes du droit de passe a notamment permis de mesurer l'intensité de la circulation routière. L'Empire ouvre à ce titre une séquence chronologique distincte, avec l'établissement d'un nouveau classement routier, l'adoption d'un autre mode de financement et la relance d'une vaste politique de construction d'infrastructures et de réhabilitation des axes existants.

Si les transports sous la Révolution ont suscité des recherches diverses, portant aussi bien sur les infrastructures que sur les moyens de communication, nombre de données conjoncturelles échappent cependant à l'investigation. Il faut reconnaître que la documentation lacunaire et dispersée ne se prête pas facilement à l'enquête historique, notamment pour la période révolutionnaire. Par ailleurs, dans la mesure où le marché des transports reste extrêmement segmenté, qu'il s'agisse des modes de circulation (navigation fluviale, flottage ou circulation routière) ou des types de trafics (marchandises, voyageurs ou courrier), ces différents

(1) Guy Arbellot, Bernard Lepetit, Jacques Bertrand, Atlas de la Révolution française, t. 1 : Routes et communications, Paris, Éditions de l'EHESS, 1987.

(2) Jean Ретот, Histoire de l'administration des Ponts et Chaussées, 1599-1815, Paris, Marcel Rivière, 1958 ; André BRUNOT, « L'administration des Ponts et Chaussées pendant la Révolution », dans L'Administration de la France sous la Révolution, Genève, Droz, 1992, p. 179-192 ; Antoine Picon, L'Invention de l'ingénieur moderne. L'École des Ponts et Chaussées 1747-1851, Paris, Presses de l'ENPC, 1992.

(3) $C f$. Jean-Marcel Goger, « La politique routière en France de 1716 à 1815 », thèse sous la direction de J.-C. Perrot, EHESS, 1988, 5 vol. ; Guy Arbellot, « Les barrières de l'An VII », Annales ESC, 30-4, 1975, p. 745-772 ; Franck LeBEAU, « Comptes et recettes des barrières établies dans l'Aisne (an VII) », mémoire de maîtrise sous la direction de Jean-Claude Perrot, Université Paris 1, 1979. 
secteurs ont fait l'objet de monographies distinctes. Si le service postal a également fait l'objet de travaux récents ${ }^{4}$, des recherches restent à mener sur d'autres entreprises de transports, comme les convois militaires ${ }^{5}$, les déplacements intra-urbains ${ }^{6}$ ou encore le flottage. D'autre part, l'attention s'est portée principalement sur l'offre de transports (investissements d'infrastructures et opérateurs de transport), en considérant le plus souvent la demande et les arbitrages qui l'orientent comme autant de facteurs découlant implicitement de l'offre.

Rendre compte de l'évolution globale des transports s'avère donc difficile. Étudier l'économie des transports suppose encore de prendre en compte son inscription dans la conjoncture générale, et de mettre en relation le rythme des flux avec les fluctuations agricoles et industrielles de la période. Y a-t-il diminution du volume de circulation marchande et du nombre de voyages? Dans quelle mesure la détérioration des infrastructures et les difficultés auxquelles fut confronté le secteur des transports peuvent-ils expliquer le repli économique de la France pendant ces années ? L'analyse des mouvements conjoncturels se prête également à une analyse spatiale des phénomènes économiques'. Quel a été l'impact de la situation des transports sur la dynamique des marchés à cette époque ? Observe-t-on un déplacement des axes de trafics en raison des troubles qui secouent certaines régions? Autant de pistes de réflexion qui restent pour l'heure à l'état de questionnements et qui mériteraient des études approfondies.

Notre propos se limitera à comprendre les implications que les choix politiques en matière de transport peuvent avoir sur la structuration de l'espace. On ne traitera pas ici du cabotage qui, outre le fait qu'il n'était pas pris en compte dans les tableaux du Maximum, voit son rôle diminuer

(4) Olivia LangloIs, « Contribution à l'histoire du service public postal (de la Révolution au tournant libéral du Second Empire) », Université de Strasbourg, thèse d'histoire du droit, sous la direction de Jean-Michel Poughon, 2003 ; Patrick Marchand, Les Maîtres de poste et le transport public en France 1700-1850, Paris, Belin, 2006 ; Anne Bretagnolle, Nicolas Verdier, « Expanding the network of postal routes in France, 1708-1833 », in Muriel LE Roux (éd.), Postes d'Europe, $X V I I I^{e}-X X I^{e}$ siècles/Post Offices in Europe, 18th-21st Century, Paris, Comité pour l'histoire de la Poste, 2007, p. 207-220.

(5) Jean-Paul Bertaud, « Contribution à l'étude des transports militaires dans les Pyrénées (1794-1795) ", 94e congrès national des sociétés savantes, Pau (1969), Paris, Bibliothèque nationale, 1971 , p. 201-218.

(6) Anne Conchon, « Paris et les transports sous la Révolution », in Raymonde MonNieR (éd.), A Paris sous la Révolution. Nouvelles approches de la ville, Paris, Publications de la Sorbonne, 2008, p. 105-116.

(7) Cf. Patrice Bourdelais, Bernard Lepetit (éd.), « Histoire et espace », Espace, jeux et enjeux, Paris, Fayard, 1986. 
dans les échanges intérieurs. Florissant jusqu'en 1793, il décline durablement pendant les guerres révolutionnaires alors que les côtes sont menacées par les flottes ennemies ; une part du trafic, qui reste toutefois à apprécier, s'est alors reportée sur les liaisons routières ou fluviales. Par de nombreux aspects, la Révolution s'inscrit dans la continuité des enjeux soulevés par la monarchie éclairée (police du roulage, question du financement des infrastructures, etc.). Dans le prolongement de l'Ancien Régime, cette période témoigne d'une volonté politique de repenser l'architecture du territoire en gommant autant que possible les discontinuités et en réduisant les disparités régionales. Il s'agira toutefois de saisir dans quelle mesure la Révolution marque une inflexion politique dans la construction de l'unité nationale avec le projet d'asseoir celle-ci et de la conforter à travers l'unification du territoire par les transports.

\section{Aménager un réseau de transports : les limites d'une ambition politique}

À partir de la géographie routière à la fois inégale sur le territoire et fortement centralisée, et des communications fluviales aménagées par la monarchie au cours du XVIII ${ }^{\mathrm{e}}$ siècle, les autorités révolutionnaires, conscientes du rôle central des transports dans le fonctionnement de l'économie et du pouvoir structurant des tracés comme des coûts, vont promouvoir la construction d'un réseau cohérent, articulé et unifié, censé rapprocher les hommes et les contrées. Celui-ci ne sera pourtant effectif qu'à l'aube du XIX'e siècle 8 .

Sous la Révolution, l'activité des publicistes et des députés, sensibles pour une majorité d'entre eux jusqu'en 1792 aux thèses physiocratiques, s'inscrit dans la continuité de l'économie politique du XVIII' siècle qui avait placé la circulation au cœur de sa réflexion, qu'il s'agisse de la mobilité des hommes et de l'acheminement des produits au même titre que le transfert d'argent ${ }^{9}$. Les discours et les mémoires réaffirment le rôle essentiel

(8) Bernard LePETIT, Chemins de terre et voies d'eau : réseaux de transport et organisation de l'espace en France, 1740-1840, Paris, Éditions de l'EHESS, 1984 ; Alain LAMOTTE, « Réseau de transport et réseau urbain dans le département du Loiret 1700-1840 », maîtrise sous la direction de Jean-Claude Perrot, Université Paris I (IHES), 1985 ; Ziba ArbeLLot, « Représentation cartographique des services publics de transports de voyageurs dans la France de 1789 », thèse $3^{\text {e }}$ cycle, Université Paris 7, 1972.

(9) Cf. Nicolas-François CAnard, Principes d'économie politique, Paris, an X ; Gilbert Faccarello et Philippe Steiner (éd.), La pensée économique pendant la Révolution française, Grenoble, Presses universitaires de Grenoble, 1991. 
des transports comme vecteurs de l'espace marchand et comme canaux de la richesse : «La circulation est le mobile de l'aisance. Mais sans de bons chemins destinés à faciliter le transport des denrées, ce grand ressort n'existe pas $\gg{ }^{10}$. Tous les auteurs sont d'accord pour reconnaître que la facilité des communications est nécessaire à l'essor des échanges marchands et à l'enrichissement du royaume : elle permet la circulation des grains et la valorisation des productions agricoles, forestières ou minières ${ }^{11}$; le développement des transports marchands doit également favoriser l'égalisation des prix dans l'espace grâce à l'interdépendance entre les régions excédentaires et celles qui sont déficitaires. À ce titre, l'économie politique de la Révolution est loin d'innover. Depuis l'Ancien Régime l'aménagement des voies de communication n'a cessé de constituer une préoccupation majeure pour les autorités centrales et locales. Outre que la fluidité de la circulation est présentée comme un levier de la prospérité, elle doit également faire diminuer les prix à la consommation et contribuer à leur uniformisation grâce au désenclavement de certaines régions.

Une question lancinante au XVIII ${ }^{\mathrm{e}}$ siècle reste par ailleurs au cœur du débat sur la politique des transports sous la Révolution : faut-il construire des routes ou améliorer le réseau navigable ? L'avantage comparatif de la voie d'eau est réaffirmé par les auteurs de l'époque. La majorité d'entre eux s'accordent sur les bénéfices de la navigation intérieure et insistent sur les possibilités d'amélioration des voies d'eau du royaume. Pour Lequinio, « l'utilité des canaux est si évidente, que l'on doit tout tenter pour arriver à les multiplier autant que les grandes routes ${ }^{12}$. Leurs arguments sont les mêmes que ceux développés tout au long du XVIII ${ }^{\mathrm{e}}$ siècle : alors que les transports routiers contribuent à renchérir le prix des grains, la navigation fluviale permet à l'agriculture de bénéficier de davantage de chevaux et de diminuer les dépenses d'entretien sur les liaisons routières ${ }^{13}$. L'ingénieur Jean-Antoine Favre va même jusqu'à comparer les

(10) Lettre circulaire aux administrations centrales du 25 thermidor an VI (12 août 1798), Recueil des lettres circulaires, instructions, programmes, discours et autres actes publics émanés du citoyen François de Neufchâteau pendant ses deux exercices du ministère de l'Intérieur, Paris, Impr. nat., an VII, t. 1, p. 58.

(11) Archives nationales, CHAN, Paris (désormais AN), F ${ }^{14}$ 607B. Mémoire de M. Cuedic de Marseille (an I) : « Des terrains encore incultes seraient bientôt couverts de moissons, de vignobles, de futaye ou changés en prairies, dans tous les environs des contrées que traverseraient ces canaux. Des bois qui dépérissent sur pied, que les frais de transport rendent sans valeur, viendraient enrichir les chantiers des ports de la nation »).

(12) J. M. Lequinio, Richesse de la République, Paris, 1792, p. 15.

(13) Cf. J.-B. MARRAGON, Rapport et projet de décret sur la navigation générale et intérieure de la République, 24 fructidor an III, p. 7-8. 
coûts du roulage et du halage en déterminant mathématiquement le nombre optimal de chevaux à utiliser ${ }^{14}$. La dégradation accélérée du réseau routier au cours de la Révolution incitera cependant à revaloriser le rôle du transport fluvial dans un État dont sont salués le nombre et l'harmonieuse distribution des cours d'eau. Rares en revanche sont les auteurs qui posent la délicate articulation entre l'espace des transports et la répartition géographique des phénomènes économiques. Si, globalement, les débats politiques sur l'intérêt des transports ne révèlent aucune originalité particulière, le calcul économique développé au même moment par les ingénieurs pour démontrer l'utilité d'une nouvelle infrastructure marque une nette évolution. S'appuyant de plus en plus sur des considérations techniques propres à la science de l'ingénieur, il s'enrichit de formules mathématiques capables de déterminer le coût d'utilisation d'une infrastructure et le meilleur moyen de minimiser les frais de transport ${ }^{15}$.

Si les préoccupations économiques sont déterminantes, les enjeux politiques sont loin d'être négligeables. Avec une verve lyrique évidente, le Directoire présente les moyens de correspondance et de communication comme "les ressorts de la puissance » de la République comparée à Hercule ${ }^{16}$. Les routes revêtent en effet une importance stratégique pour les convois militaires en direction des frontières, l'acheminement des subsistances pour les troupes et le ravitaillement des grandes villes, notamment de la capitale. En mars 1793, Charles Barbaroux propose de construire un vaste ensemble de canaux à des fins autant politiques qu'économiques ${ }^{17}$ : il s'agissait non seulement de garantir la « félicité publique » par la fluidité des échanges mais aussi de fournir du travail aux chômeurs, aux soldats démobilisés et aux vagabonds. Un mémoire renvoyé par le Comité de Salut public au Comité des Ponts et Chaussées prétendait terminer la guerre de Vendée en coupant la région par de grandes routes. Et de fait, l'équipement routier du département de la Vendée fut poursuivi alors que partout ailleurs les constructions ont été quasiment stoppées entre 1790 et

(14) Essai sur la théorie des torrens et des rivières, contenant les moyens les plus simples d'en empêcher les ravages, d'en rétrécir le lit et d'en faciliter la navigation, le hallage et la flottaison ; accompagné d'une discussion sur la navigation intérieure de la France..., Paris, Bidault, an $\mathrm{V}$.

(15) François Etner, « Le calcul économique, 1789-1815 », dans Gilbert FACCARELlo et Philippe Steiner (éd.), La pensée économique..., op. cit., p. 351-361 ; François ETner, Histoire du calcul économique en France, Paris, Economica, 1987.

(16) AN, 1A 11 : Bulletin des lois $n^{\circ} 164$; Proclamation du Directoire exécutif du 21 frimaire an VI relatif à la réparation des routes.

(17) De l'influence de la guerre maritime sur le commerce, et de l'organisation des travaux publics, Paris, Impr. nat., 1793. 
$1810^{18}$. De façon plus générale, les transports sont présentés comme le meilleur gage de la cohésion nationale et « le moyen le plus puissant pour entretenir cette correspondance active qui lie ensemble toutes les opérations d'un grand gouvernement $»^{19}$.

Des projets multiples, proposant des innovations techniques (construction des ponts, machines pour remonter les bateaux, etc.) ou suggérant l'aménagement d'un nouvel axe de circulation, affluent sur les bureaux des comités de la Constituante et de la Législative. Pour les travaux à exécuter à proximité des frontières, l'examen des propositions relève d'une assemblée mixte composée de commissaires de l'assemblée des Ponts et Chaussées et du corps du Génie. À compter du mois de février 1793, les affaires concernant les infrastructures sont remises à un comité spécialisé des Ponts et Chaussées après avoir été pendant plus de trois ans du ressort du comité à l'agriculture et au commerce. Le discours de Barère du 21 ventôse an II convainc le Comité de Salut public de créer une commission nationale de trois membres nommés par la Convention pour examiner les projets, superviser la construction comme l'entretien des infrastructures, et passer les marchés. Cette institution n'a toutefois qu'une existence éphémère. Après thermidor an II, l'ancien Comité des Ponts et Chaussées devient le Comité des Travaux publics. Outre la première section responsable des communications routières et fluviales, celui-ci a également en charge les bâtiments civils et les fortifications. Le registre des délibérations de ce comité se révèle extrêmement instructif pour saisir la politique des transports menée par la Convention ${ }^{20}$. Pour l'essentiel, les affaires rapportées concernent le difficile entretien des axes routiers, les pertes que subissent les entrepreneurs comme les transporteurs, ainsi que les carrières des ingénieurs. De nombreux projets de canaux, émanant de particuliers comme de sociétés populaires locales, sont soumis à cette autorité ${ }^{21}$. Ils rendent compte du nombre des initiatives dispersées en faveur de l'amélioration du réseau routier et des voies navigables.

Poursuivant la politique engagée par la monarchie administrative, l'État révolutionnaire entend investir l'espace national en son entier et

(18) Bernard LePETIT, Chemins de terre, op. cit., p. 76.

(19) Conseil des Cinq-Cents. Rapport et projet de résolution au nom d'une commission spéciale par J.-B.-J. Borel-Vernière, député par le département de la Haute-Loire, sur la navigation fluviale (28 frimaire an VII), p. 2.

(20) AN, AF II* 34-35. Procès-verbaux (1793-an IV).

(21) Plusieurs de ces projets sont conservés sous les cotes AN, $F^{12} 1515, F^{14} 708 \mathrm{~A}$ et AD XIII 11,12 et 15 . 
assurer une couverture complète du territoire. À l'échelle de la République, deux comités de la Convention se partagent pour l'essentiel les compétences en matière de transport : au Comité des Travaux publics revient le soin de construire et d'entretenir les infrastructures, tandis qu'un Comité des Postes, transports et messageries traite de tout ce qui concerne le trafic ${ }^{22}$. La première section de ce bureau a en charge les messageries nationales, le roulage et les voitures par terre ; les trois autres s'occupent respectivement de la navigation intérieure, de la poste aux lettres et de la poste aux chevaux. Dans le même temps, le maillage des bureaux de poste continue de s'étendre sous la Révolution. Les nombreuses enquêtes réalisées au cours de la période témoignent également de cette ambition nationale ${ }^{23}$. En juin 1790, l'Assemblée constituante somme les directoires des départements de faire dresser le tableau des routes et des canaux de leur circonscription ${ }^{24}$. Un arrêté du Comité de Salut public daté du 12 messidor an II ordonne entre autres choses le recensement des routes, des canaux et des rivières navigables sur l'ensemble du territoire. Au début du mois de mars 1794, le Comité des Ponts et Chaussées demande à l'ingénieur en chef de chaque département de fournir une note sur les canaux en activité, commencés ou projetés ${ }^{25}$. Les réponses à ces enquêtes, outre leur parcimonie, ne fournissent toutefois pas de renseignements homogènes sur les infrastructures et pour un grand nombre ne reprennent pas le classement hiérarchisé des voies élaboré par la monarchie.

Dès les premières années de la Révolution, de nombreux mémoires dressent un inventaire des canaux à aménager dans le cadre d'un réseau cohérent et articulé de navigation ${ }^{26}$. De la section «Navigation » du Comité

(22) AN, AF II*36-38. Procès-verbaux (fructidor an II-an III).

(23) Pierre CARON, «Une enquête sur l'état des routes, rivières et canaux au début de l'an II », Bulletin d'histoire économique de la Révolution, 1917-1919, Paris, p. 1-362. Georges BourgIN, Mémoires et documents. IX. Fragments d'une enquête économique du ministre Roland auprès des ingénieurs des Ponts et Chaussées (1792-1793), Besançon, Imp. Jacques et Demontrond, 1942. Cf. Isabelle GUÉGAN, « Inventaire des enquêtes administratives et statistiques 1789-1795 », maîtrise sous la direction de Michel Vovelle, Université Paris 1 (IHRF), 1988, p. 209-218. Bernard LePETIT, Chemins de terre, op. cit., p. 28-29.

(24) Procès-verbaux de l'Assemblée constituante (28 juin 1790 et 30 juin 1790).

(25) Augustin Cochin, Charles Charpentier, Les actes du gouvernement révolutionnaire, Paris, Librairie Alphonse Picard et fils, 1920-1935, t. 2, p. 365.

(26) $\mathrm{AN}, \mathrm{F}^{14} 607 \mathrm{~B}$ : «État des ouvrages, plans, nivellemens, devis, détails et mémoires sur les différens canaux et jonction de rivières à rendre navigables et autres par le $\mathrm{S}$. Clavaux ingénieur et capitaine des dragons ") ; J.-M. Lequinio, Richesse de la République, Paris, 1792 ; A. Hageau, Avantage démontré d'un système général de navigation dans l'intérieur de la République (an III) ; RAUCH, Vues nautiques pour la navigation intérieure (an III), etc. 
des Travaux publics à la création par François de Neufchâteau en l'an VII d'une division de la navigation intérieure (la sixième au Ministère de l'Intérieur) ${ }^{27}$, la volonté politique est la même de centraliser la somme des connaissances accumulées sur les cours d'eau depuis l'Ancien Régime. Le " système général de navigation intérieure » auquel travaille J.-B. Marragon à partir de l'an II revêt le même objectif de " prévenir le défaut de concordance et d'ensemble qui résulterait de projets détachés et locaux. C'est s'assurer d'un moyen exact d'apprécier tous les projets qu'on a présentés ou qu'on pourra présenter au gouvernement et de n'adopter que ceux qui peuvent concourir au système général $»^{28}$. Dans le même temps, Dupain-Triel, après avoir présenté une première carte de la navigation intérieure en octobre 1789 au Comité d'Agriculture et de commerce, travaille à une seconde version conforme au cadre départemental ${ }^{29}$. Une «Agence pour la navigation intérieure » est créée le 10 messidor an III pour surveiller le mouvement général des coches, diligences et barques en état de naviguer sur tous les cours d'eau de la République ${ }^{30}$. En ventôse an VI, le Directoire ordonne la visite des rivières et des canaux pour inventorier les obstacles à la navigation ${ }^{31}$, et le ministre de l'Intérieur forme le projet de réunir douze commissions chargées d'évaluer l'utilité de nouveaux canaux à ouvrir dans le cadre du « système général de navigation intérieure $\|^{32}$. Le projet de relier le Rhin au Rhône, déjà soumis à la monarchie dans les années 1750 et réactivé dès les premières années de la Révolution, doit même permettre dans l'esprit de ses promoteurs de penser le réseau de navigation fluviale à une échelle européenne ${ }^{33}$.

Si la navigation fluviale se prête aisément à une réflexion globale en termes de réseau, on retrouve une volonté similaire d'appréhender l'aménagement routier à l'échelle de la République. En 1792, P.-J. Planier,

(27) Cf. Dominique Margairaz, François de Neufchâteau. Essai de biographie intellectuelle, Paris, Publications de la Sorbonne, 2005, p. 289-295.

(28) AN, AF II* 34-35. Séance du 7 floréal an III.

(29) $\mathrm{AN}, \mathrm{F}^{14} 607 \mathrm{~B}$.

(30) AN, $\mathrm{F}^{12} 1515$.

(31) Recueil des lettres [...] du citoyen François de Neufchâteau, an VII, t. 2, p. 17 : Circulaire du ministre de l'Intérieur aux administrations centrales de départements, aux commissaires du Directoire exécutif et aux ingénieurs en chef.

(32) Ibidem, t. 1, p. 336-350 : Circulaire du 23 frimaire an VII ; AN, AD XIII 15 : Plan des commissions qui vont être établies pour la navigation intérieure (an VII).

(33) AN, AD XIII 11 : Prospectus d'un canal de navigation de 25 lieues de longueur qui, en liant la jonction du Rhône et de la Saône à la Loire, avec celle du Rhin au Danube et autres fleuves intermédiaires, feroit communiquer, par les frontières de l'Alsace et de la Franche-Comté, toutes les mers qui environnent l'Europe (1790). 
ingénieur des Ponts et Chaussées, répond dans ce sens à l'enquête diligentée par J.-M. Roland : " Il serait encore plus à désirer qu'il y eût un projet général de l'itinéraire de la France arrêté par les départements, qui fût à l'abri des variations et des changements des corps administratifs $»\rangle^{34}$.

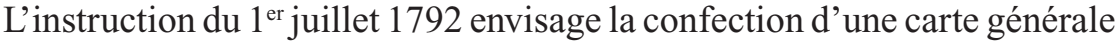
des routes de la République ${ }^{35}$. En germinal an III, un projet de système général des routes est soumis au Comité des Travaux publics ${ }^{36}$. Il n'en reste pas moins que des considérations locales l'emportent bien souvent sur l'inscription de telle desserte routière dans le projet de réseau mûri par l'administration centrale.

La Révolution marque à cet égard une étape essentielle pour la promotion d'un réseau (ou " système ») national et coordonné de transports, même si le concept de « réseau » s'impose plus tardivement ${ }^{37}$. L'administration s'efforce alors de concevoir les routes et les voies navigables dans le cadre d'un système articulé de circulation. Dans un mémoire intitulé Des travaux publics, qu'il soumet en 1790 au Comité d'Agriculture et de commerce, l'ingénieur en chef de Caen Armand Lefebvre plaide pour une interconnexion des liaisons routières avec les rivières et les canaux. E.-Cl. Marivetz et son Plan d'un nouveau mode pour l'administration des Ponts et Chaussées de France (1790), qui reprend en fait le Système général, physique et économique des navigations naturelles et artificielles à l'intérieur de la France (1788), vise à « régénérer l'Empire français par un plan [...] de la navigation intérieure du royaume, coordonné avec les routes de terre ». L'ingénieur des Ponts et Chaussées du Loiret développe la même idée dans la réponse qu'il fait à l'enquête du ministre de l'Intérieur Roland en l'an II : « La navigation, comme les routes, doivent avoir entre elles une corrélation combinée d'après leur utilité locale et surtout d'après leur utilité générale ». En l'an III, le Comité des Travaux publics décide la création d'une section «sistématique pour les lois à proposer sur les routes, ponts, canaux, rivières, \&c $\|^{38}$. Afin d'accélérer la réparation des infrastructures, un projet de décret du Comité

(34) Georges Bourgin, Fragments d'une enquête économique..., op. cit., p. 98.

(35) Bernard LePetit, Chemins de terre ..., op. cit., p. 28 . AN, $F^{14} 954$ (2): Instruction sur la carte itinéraire que chaque ingénieur en chef doit fournir de son département (1792).

(36) AN, AF II* 34-35. Séance du 11 germinal an III.

(37) Cf. Bernard LePetit, "L'impensable réseau » et André Guillerme, " L'émergence du concept de réseau 1820-1830 », dans Gabriel Dupuy, Réseaux territoriaux, Caen, Paradigme, 1988, p. 21-32 et 33-50.

(38) Georges Bourgin, Fragments d'une enquête économique..., op. cit., p. 120 ; et AN, AF II*34-35. Séance du 22 germinal an III. 
de Salut public prévoit la division du territoire en dix arrondissements, chacun étant placé sous la responsabilité d'un représentant du peuple. Ce projet ne semble pourtant pas avoir eu de suite. Ce plan d'un « système général des transports intérieurs » court ainsi tout au long de la Révolution jusqu'au plan de cartographie des routes et des cours d'eau impulsé en l'an VII par le ministre de l'Intérieur mais demeuré faute de temps à l'état de projet $^{39}$.

L'esprit de système que fait valoir la Révolution peine toutefois à dissimuler la réalité de l'espace des transports caractérisé par de fortes disparités d'équipement à l'échelle nationale et des discontinuités dans les liaisons. La période révolutionnaire marque assurément un temps d'arrêt pour la construction des routes, et de déshérence pour leur entretien. Certains investissements proposés pour garantir une meilleure cohésion territoriale et permettre le désenclavement de certaines régions se seraient sans doute, faute d'une évaluation rigoureuse de la demande, révélés d'une faible rentabilité. Même si les autorités tentent tant bien que mal de poursuivre l'extension des liaisons fluviales, les résultats, pour le moins modestes, s'expliquent en partie par la baisse des crédits d'équipement : en un an à compter d'octobre 1792, les comités de la Convention examinent 23 projets de canaux et d'aménagement de rivières ; l'année suivante le nombre de soumissions est inférieur à dix ${ }^{40}$. Après dix ans de travaux, le canal du Centre est mis en eau en 1793. En revanche, le chantier du canal du Nivernais reliant la Loire et l'Yonne, lancé par la monarchie, est arrêté au printemps 1791. Plusieurs concessions de canaux - entre la Loire et la Seine par l'Essonne (1791), de Sommevoire (HauteMarne) à l'Aube, pour relier l'Ille à la Rance (1792), entre l'Eure et le Loir (1793) et dans la partie amont du lit du Rhône (an IV) - avortent faute de crédits suffisants pour mener à bien des chantiers souvent coûteux ou hasardeux ${ }^{41}$. Le canal de la Sambre à l'Oise, dont la construction a été entérinée, reste à l'état d'études préparatoires. Les travaux du canal de Bourgogne sont interrompus en 1794. Pour l'essentiel, les travaux suspendus pendant la Révolution ne seront relancés que sous l'Empire. Le pouvoir napoléonien va toutefois préférer, à l'organisation d'un réseau

(39) Dominique Margairaz, «La géographie des administrateurs », in Hélène Blais, Isabelle Lagoulais (éd.), Géographies plurielles. Les sciences géographiques au moment de l'émergence des sciences humaines (1750-1850), Paris, L'Harmattan, 2006, p. 185-216 (en particulier p. 206-208).

(40) Jean-Claude Perrot, «Voies nouvelles pour l'histoire économique de la Révolution », $A H R F, \mathrm{n}^{\circ} 219$, janvier-mars 1975, p. 41.

(41) AN, AD XIII 11. 
ramifié à l'échelle nationale, la concentration des investissements sur quelques axes principaux ${ }^{42}$.

La Révolution, en réalisant l'unification du territoire et en proclamant l'unité comme l'indivisibilité de la République, s'est ainsi attachée à penser le territoire comme un tout innervé par les communications et structuré par une logique réticulaire. Des débats parlementaires se dégagent bien une typologie hiérarchisée des lignes de transport et l'idée d'un système articulé. Cependant, outre l'impécuniosité d'un État incapable de réaliser ses ambitions en matière d'équipement, il y a un évident hiatus entre la dimension nationale du projet de circulation et les échelles auxquelles sont menés les chantiers.

\section{Les dénivellations régionales des prix de transport}

Depuis l'ouvrage pionnier d'André Rémond, la question des prix et des coûts du transport n'a guère retenu l'attention des historiens ${ }^{43}$. Il s'agit pourtant là d'un chantier de recherche fondamental pour mesurer la place des transports dans l'activité économique. L'étude de ces coûts suppose de prendre en considération plusieurs variables, à commencer par la distance des trajets et la vitesse de circulation. Il convient aussi de se demander dans quelle mesure le déséquilibre permanent entre l'offre et la demande sur un grand nombre d'itinéraires et les disparités structurelles des flux aller et retour pèsent sur les prix de transport. Sous la Révolution, sur fond de crise économique et sociale, les transporteurs professionnels ne cessent de se plaindre des difficultés qu'ils rencontrent dans leur activité $^{44}$. Les demandes d'indemnité de la part des voituriers et des maîtres de poste se multiplient pour compenser la hausse des prix. Les difficultés conjoncturelles, aggravées à compter de 1792 par les besoins de la guerre, ont indéniablement pesé sur l'économie des transports : l'inflation des fourrages et la pénurie de chevaux ont ainsi contribué à renchérir notablement les frais d'exploitation. Confrontés à ces surcoûts, les entrepreneurs de messagerie réclament des indemnités voire la résiliation de leurs baux. Il est un fait que le coût de transport se révèle moins élastique que les autres prix et a tendance à s'alourdir en période de dépression ${ }^{45}$. À ce titre,

(42) Bernard LePETIT, Chemins de terre ..., op. cit., p. 119.

(43) André RÉmond, Études sur la circulation marchande, en France aux XVIII ${ }^{\mathrm{e}}-X I X^{\mathrm{e}}$ siècles, t. 1 : Prix des transports marchands de la Révolution à l'Empire, Paris, M. Rivière et C $\mathrm{C}^{\mathrm{ie}}, 1956$.

(44) Richard Совв, « Le mouvement revendicatif parmi les bateliers de l'Oise et Marne au cours de l'hiver 1793-1794», Revue d'histoire économique et sociale, 32/4, 1954, p. 353-366.

(45) Cf. Daniel L'Huillier, Le coût de transport, Paris, Éditions Cujas, 1965. 
une des interrogations formulées en 1975 par Jean-Claude Perrot reste d'actualité : la crise des échanges pendant cette période a-t-elle freiné la spécialisation régionale de la production et entraîné une plus grande dispersion géographique des prix ${ }^{46}$ ? Des analyses statistiques comme celles qui ont mesuré l'intégration des marchés du blé devraient sans doute être étendues à d'autres produits ${ }^{47}$.

En matière de transports, comme pour les autres secteurs économiques, la politique des assemblées révolutionnaires hésite entre une ferme volonté de libéraliser le marché des services de transports et un dirigisme évident, notamment à l'époque de la Convention. La question de l'incidence de ces choix sur la géographie des prix reste entière : l'ouverture à la concurrence du transport des voyageurs a-t-elle contribué à faire baisser les prix des courses et à atténuer leurs disparités ? Alors que le roulage était déjà une activité libre sous l'Ancien Régime de même que la batellerie à quelques exceptions près (comme celles des gribanniers de la Somme et des belandriers des cours d'eau septentrionaux), l'ouverture à la concurrence concerne surtout le transport des voyageurs et des petits paquets. La loi du 29 août 1790 commence par abolir le privilège des messageries qui jusque-là assuraient le transport des voyageurs, des métaux précieux et des paquets de faible poids. Cette libéralisation est toutefois soumise à certaines conditions restrictives : les voituriers qui souhaitent entreprendre ce type de courses doivent faire préalablement une déclaration dans les huit premiers jours de chaque année au greffe de la municipalité du lieu de leur domicile et la renouveler chaque année s'ils veulent poursuivre leur activité. Symétriquement, les messageries conservent le monopole de la régularité des services pour le transport des voyageurs avec des départs à jour et heure fixes, et peuvent seules exploiter des relais. Le prix de la course est convenu de gré à gré mais ne doit pas excéder les tarifs édictés en 1775 . Une dimension particulière de la compétition dans les transports tient en effet non pas à une concurrence par les prix, mais à la qualité de service et à l'enjeu des horaires. Il faut attendre la loi 9 vendémiaire an VI (30 septembre 1797) qui supprime la Régie des Messageries nationales, pour que ce service s'ouvre pleinement à la libre entreprise. Désormais tout particulier peut conduire ou faire conduire librement des voyageurs et des ballots. L'État impose toutefois un droit s'élevant à un dixième du prix des places pour l'exploitation de

(46) Jean-Claude PerRot, « Voies nouvelles... », art. cit., p. 42.

(47) Les travaux menés dans le cadre de l'école labroussienne sur les dénivellations des prix en France ont surtout traité des grains. 
voitures à horaires réglés, et une patente pour les autres services. Tandis que l'entreprise des messageries nationales est démantelée et libéralisée, le monopole d'État est quant à lui réaffirmé sur la poste aux lettres. À une autre échelle, la libéralisation des transports publics à Paris implique dès 1789 la disparition des entreprises privilégiées qui jusque-là encadraient les services de transports. Quiconque dispose d'une voiture peut désormais se livrer à cette activité à condition de se soumettre aux règles de police et de s'acquitter d'une taxe de cinq sols par jour et par véhicule ${ }^{48}$. Quelle a été l'évolution des prix face aux nouvelles conditions de concurrence ? Assiste-t-on dans un premier temps à une baisse des prix liée notamment à la multiplication du nombre des opérateurs ? La reconfiguration de l'offre de transports, avec la faillite des entreprises les plus fragiles et la recherche d'économies d'échelles dans les services publics d'acheminement ${ }^{49}$, a-t-elle permis par la suite une stabilisation des prix ? À l'évidence, l'ouverture à la concurrence ne doit pas se limiter aux seules conséquences en termes de prix ; elle revêt d'autres implications notamment spatiales, comme l'abandon éventuel de certaines dessertes jugées peu rentables.

Alors même que la Révolution consacre la fin des privilèges en matière de services de transports, qu'il s'agisse des carrosses parisiens et des messageries, la Convention impose des mesures radicales de réquisition. À l'automne 1792, les autorités décrètent le recensement des montures et des voitures ${ }^{50}$. Il s'agit en priorité de pourvoir aux besoins des armées de la République et de satisfaire aux impératifs d'approvisionnement de la capitale comme des principales villes. Les réquisitions, auxquelles échappe toutefois le service des messageries ${ }^{51}$, contribuèrent indéniablement à renchérir les coûts du roulage. La navigation fluviale fait également l'objet d'enquêtes répétées en vue de la mobilisation économique qu'impose la conjoncture militaire et frumentaire. Le 26 brumaire an II (16 novembre 1793), la commission des subsistances lance une vaste consultation auprès des districts pour connaitre les moyens de la batellerie (nombre de bateaux en circulation par rivière, adresse de leur

(48) $\mathrm{AN}, \mathrm{AD}$ I 88 : Loi relative à la résiliation du privilège exclusif des carrosses de place et des voitures des environs de Paris (24 novembre 1790).

(49) $C f$. Anne Conchon, « Postes et messageries en France (milieu du XVII ${ }^{\mathrm{e}}$ s.-fin XVIII ${ }^{\mathrm{e}} \mathrm{s}$.) : prix et économie du transport des lettres », dans Muriel Leroux (éd.), op. cit., p. 221-233.

(50) Procès-verbaux de l'Assemblée Nationale, Paris, (2 septembre 1792). Les voitures de pur agrément devaient être réquisitionnées au profit des municipalités.

(51) Procès-verbaux de l'Assemblée constituante (28 juillet 1791). 
propriétaire, ports de chargement et produits habituellement transportés) ${ }^{52}$. L'année suivante, en vertu d'un arrêté du Comité de Salut public du 15 messidor an II (3 juillet 1794), les détenteurs de coches ou de bateaux, ainsi que tous les citoyens attachés au service de la navigation fluviale (aides de pont, garçons de bateaux, haleurs...) doivent se faire enregistrer auprès des municipalités ${ }^{53}$. Il s'agit pour les autorités non seulement de recenser les moyens de transports, mais aussi d'assurer à ce secteur d'activité un nombre de préposés suffisant, et éviter une pression sur les salaires qui ne manquerait pas de se répercuter sur les coûts de transport et les prix à la consommation. Bateliers et postillons échappent ainsi à la conscription; de même les ouvriers employés aux travaux publics sont dispensés de service militaire ${ }^{54}$.

De façon générale, les prix de transport constituent un des facteurs de la diversité régionale des prix. C'est encore plus vrai en période de crise quand l'inflation et les réquisitions contribuent à accentuer ces inégalités ${ }^{55}$. Il est toutefois quasiment impossible de suivre année par année les éléments - prix des fourrages et salaires notamment - qui renchérissent le coût de transport. En outre, la dégradation des infrastructures a sans doute accéléré l'usure des véhicules comme le renouvellement des animaux de trait, de même qu'elle a pu contraindre les transporteurs à réduire la charge utile.

C'est précisément dans ce contexte que la Convention se décide à réglementer les prix de transports marchands dans le cadre du Maximum des prix et des salaires. L'enjeu de cette mesure est de fixer les prix des transports afin de déterminer le prix de vente des produits, et de freiner une hausse tendancielle des coûts d'acheminement, plus ou moins accusée selon les régions. Quand il existe une concurrence possible entre la voie d'eau et la route, les agents nationaux des districts doivent indiquer le coût du transport fluvial de préférence au prix du roulage, afin de ne pas augmenter artificiellement les prix des articles acheminés par bateau.

(52) Augustin CochIn, Charles CHARPENTIER, Les actes du gouvernement révolutionnaire, op. cit., t. 1, p. 489 ; AN, F ${ }^{11} 268$.

(53) Augustin CochIn, Charles CHARPENTIER, Les actes du gouvernement révolutionnaire, op. cit., t. 3, p. 464-466 (8 juillet 1794).

(54) Procès-Verbaux de l'Assemblée Nationale (6 septembre 1792). AN, F ${ }^{12} 1515$. Arrêté (27 pluviôse an III) du représentant du peuple Porcher en mission dans les départements du Loiret, Loiret-Cher, Indre-et-Loire.

(55) Cf. Dominique Margairaz, «Dénivellations des prix et inégalités de développement régional dans la France de 1790. Essai de cartographie », $A H R F, \mathrm{n}^{\circ} 244$, avril-juin 1981, p. 272 : «Il est en tout cas plus que probable que les distorsions fortes, que 1'on enregistre entre districts voisins, sont à attribuer pour une part non négligeable au coût des transports ». 
Pour l'essentiel, seuls les échanges interrégionaux et nationaux par route ou par eau sont concernés. La circulation dans les limites du district pour le ravitaillement des marchés locaux échappe en effet au Maximum. Le taux devait différer selon le mode de circulation (par eau/par terre), le sens de navigation (remonte/avalage) et la hiérarchie des axes (grande route/chemin de traverse) ${ }^{56}$. En fait, rares sont les agents nationaux qui prennent la peine de différencier le chemin de traverse de la grande route, ou de préciser le mode de transport. Au final, c'est une taxation uniforme pour l'ensemble du territoire - par lieue et par quintal - qui est retenue, sans tenir compte des dénivellations régionales et temporelles inhérentes aux prix de transport. De nombreux paramètres peuvent en effet influer sur ceux-ci, tels que le caractère saisonnier du trafic, la nature des objets transportés ou encore des aléas divers (crues ou étiages, routes impraticables en temps de pluie, chantiers, vidange des canaux...) $)^{57}$, sans compter les coûts de transport, les dépenses de bouche et les frais d'hébergement du voiturier, qui dans le cas de transports au long cours, restent difficiles à prévoir en période de crise. Bien que les autorités révolutionnaires soient tout à fait conscientes de ces contingences, il s'agit pour le Comité des Subsistances d'établir un prix moyen. Il n'empêche que l'espace postulé par cette logique tarifaire s'ajuste mal aux contraintes réelles de la circulation marchande. Les distances sont en effet établies sur la base des itinéraires directs entre deux destinations alors même que l'on sait pertinemment que les rouliers étaient souvent amenés à faire des détours. Par ailleurs, pour la navigation fluviale, la distance devait être calculée « par le nombre de lieues de poste qu'il y a par la route de terre du lieu de départ à celui de l'arrivée $\aleph^{58}$. Cela revient de fait à introduire de notables déformations dans l'espace des échanges et a fortiori à fausser le prix des transports en étalonnant les méandres et les confluences des liaisons fluviales à l'aune des tracés rectilignes des routes de poste.

Quelles que soient les incohérences et les lacunes de cette enquête, elle permet d'esquisser une géographie des prix de transport dans les

(56) Cf. Thomas Leroux, Le commerce intérieur de la France à la fin du XVIII siècle, Paris, Nathan, 1996.

(57) Instruction sur le tableau général du Maximum du 14 ventôse an II (4 mars 1794) : «Les traités ou conventions entre les commerçants et les voituriers continueront de se faire de gré à gré sans que les prix convenus entre eux puissent augmenter ou diminuer ceux des denrées et marchandises, l'intention de la loi du 6 ventôse n'étant pas de taxer le voiturier, mais bien de fixer le prix que ne pourra pas outrepasser le marchand en vendant au consommateur $»$.

(58) Le Maximum général. Instruction, recueil de textes et notes, Paris, Imp. nat., 1907, p. 61 : loi du 6 ventôse an II (24 février 1793), art. VII. 
premières années de la Révolution. De la même façon, le Directoire puis le Consulat, en rétablissant des péages, vont s'efforcer de gommer un des facteurs d'indétermination régionale du coût de transport, en adoptant une base de tarification uniforme et progressive selon la distance des trajets.

\section{La tarification des infrastructures : une nouvelle métrique de l'espace}

Alors que la monarchie avait tenté dans les dernières décennies du XVIII ${ }^{e}$ siècle de supprimer les péages féodaux, c'est la Révolution qui va les faire disparaitre en 1790. Rapidement, l'État doit se résoudre à tarifer à nouveau l'usage des infrastructures. Si le droit de passe a fait l'objet de nombreux travaux, il n'en est pas de même pour la navigation intérieure. Proposé dès l'an VII par une commission réunie pour connaître l'état des canaux de navigation et créé par la loi du 30 floréal an X (20 mai 1802), l'octroi de navigation n'a pratiquement pas été étudié. Pour asseoir la perception de ces nouveaux péages, l'État va inventer une structuration originale de son espace.

En 1790, Chaumont de la Millière estime que les routes de première (reliant Paris aux principales villes) et de deuxième classe (liaisons provinciales) sont globalement en bon état ${ }^{59}$. Cependant, avec la suppression de la corvée à la veille de la Révolution et l'abolition des péages décrétée en 1790 , l'État se trouve rapidement confronté à de criantes difficultés financières pour entretenir les infrastructures de transport. Seul l'entretien de canaux, qu'ils aient été confisqués par la Révolution (canaux du Midi, d'Orléans, du Loing...) ou non (canal de Briare), restait couvert par la perception de droits sur la navigation. Le 15 janvier 1790, la Constituante confie aux départements le soin d'entretenir les infrastructures de leur circonscription, en les autorisant à prélever jusqu'à quatre sols additionnels sur les contributions foncière et mobilière. Cependant, la décentralisation des financements et la dilution des responsabilités se révèlent un échec, les départements se révélant incapables de rassembler les fonds nécessaires à la maintenance des infrastructures. Dès le mois d'avril 1791, la Constituante doit donc se résoudre à débloquer une série de crédits ${ }^{60}$.

(59) AN, AD XIII 16. Mémoire sur le département des Ponts et Chaussées, Paris, Imp. Royale, janvier 1790.

(60) En 1790, l'Assemblée nationale décréta un gros versement pour l'achèvement du canal de Bourgogne et décida le financement du canal du Cambrésis qui devait longer l'Escaut entre Cambrai 
À compter de 1792, avec l'entrée en guerre de la France, la question de l'entretien des voies de communication ne figure plus parmi les priorités budgétaires. Le Comité des Travaux publics multiplie les circulaires pour connaître l'état des infrastructures afin d'évaluer les fonds nécessaires aux réfections les plus urgentes. Pour activer les travaux, les administrations départementales sont autorisées à procéderà des réquisitions d'hommes, de chevaux, de voitures et de matériaux. Les réquisitions de main-d'œuvre, qui font resurgir le spectre de la corvée, se révèlent toutefois difficiles à concilier avec les levées et les travaux agricoles. La Convention y renonce en décembre 1794 pour revenir au système des adjudications. Dans une circulaire adressée à tous les directoires de départements le ministre de l'Intérieur invite « les habitants à porter momentanément le secours de leurs bras, de leurs chevaux, de leurs moyens quelconques vers les grands chemins ». L'esprit de sacrifice des habitants de la commune de Hirenek (Bas-Rhin), qui faute de chevaux s'étaient attelés eux-mêmes aux chariots nécessaires à la réparation des routes, est érigé en exemple révolutionnaire ${ }^{61}$. Administrateurs et ingénieurs ne cessent de déplorer la dégradation des infrastructures de transports. Ce sont assurément les routes qui font l'objet du constat le plus alarmiste. Aux Réflexions sur le délabrement des routes que Trippier livre le 22 janvier 1793 au Comité de Commerce de la Convention, fait écho la circulaire de François de Neufchâteau le 25 thermidor an VI (12 août 1798) sur le mauvais état du réseau routier. Les raisons en sont multiples : avec le discrédit de l'assignat, les ouvriers refusent de travailler à l'entretien des routes s'ils ne sont pas payés en numéraire ou en grains ; les adjudications de travaux trouvent difficilement preneurs à cause de la rareté et de la cherté des salaires et des voitures; le poids des convois militaires contribue par ailleurs à détériorer les revêtements des chaussées ${ }^{62}$.

Faute d'un entretien régulier, la situation des cours d'eau n'est guère plus enviable. Finalement, le décret du 16 frimaire an II ( 6 décembre 1793) retire aux départements la responsabilité de l'entretien des infrastructures. L'État s'en réserve le soin grâce à des expédients tels que la

(Suite de la note 60 page 21.)

et Manières. En janvier 1792 encore, elle débloqua les fonds nécessaires à la continuation des travaux du canal de la Saône à la Seine. Pour parer aux travaux les plus urgents sur le réseau existant, l'État accorda d'abord trois millions pour les Ponts et Chaussées en septembre 1791, puis deux millions quelques mois plus tard pour l'entretien des routes.

(61) AN, AF* II 34-35. Séance du 12 prairial an II.

(62) Adolphe GARNIER, « Les Travaux publics (routes et ponts) dans les Vosges pendant la Révolution », La Révolution dans les Vosges, 4e année, 1910-1911, p. 192-216. 
réquisition ponctuelle des militaires pour des travaux de terrassement - recours qui ne peut qu'être très limité en temps de guerre -, l'adjudication des ouvrages les plus coûteux, et la distribution de subventions sporadiques ${ }^{63}$. Mobilisés pour parer aux travaux les plus urgents, les représentants en mission multiplient les inspections des axes routiers, des voies fluviales et des entreprises de transport ${ }^{64}$.

Le financement de l'entretien du réseau d'infrastructures sur les contributions directes s'étant révélé un échec, il faut renouer avec le système des péages. À l'issue de longs débats, le Directoire se décide le 24 fructidor an V (10 septembre 1797) à percevoir une taxe d'entretien, encore appelée " droit de passe ", perçue sur les chariots employés au roulage, les voitures transportant des voyageurs, les bêtes de somme comme les montures. En attendant le placement des barrières, l'État en appelle aux souscriptions volontaires afin de trouver les subsides nécessaires à la réparation du réseau routier ${ }^{65}$. Malgré un appel vibrant au patriotisme, les fonds collectés semblent avoir été insignifiants ${ }^{66}$.

Pour déterminer l'emplacement des nouvelles barrières, plusieurs députés songent dans un premier temps à établir une trame de coordonnées neutres et géométriques. Defermont (en septembre 1796), puis Dubois (en octobre 1798) proposent de placer les barrières à des distances équivalentes pour faciliter le calcul de la taxe : de 5 à 6 lieues (soit 22 à $26 \mathrm{~km}$ ) pour le premier, et de 2 à 4 lieues $(10-20 \mathrm{~km})$ pour le second. Finalement le 28 pluviôse an VI (16 février 1798), le ministre de l'Intérieur Letourneux adresse aux administrations départementales la localisation des 1200 barrières à établir, un exemplaire de tarif et un modèle de barrière. Pour minimiser les coûts d'installation, les barrières sont pour la plupart placées aux endroits où le trafic est le plus élevé, aux intersections avec des routes de poste et les axes les plus fréquentés, ainsi qu'aux portes des villes. Symétriquement, on limite le nombre de barrières sur des axes qu'il est impossible de quitter. Ainsi sur le trajet de Vienne à Valence, dénué de

(63) En frimaire an II, 85 millions furent ainsi alloués au ministre de l'Intérieur pour financer la réparation des routes et ponts de la République.

(64) Dans le département de la Corrèze, Paganel dut approuver un arrêté qui accordait des primes aux entrepreneurs pour l'achat de subsistances distribuées aux ouvriers. En 1795, Blanqui, Fayolle, Lacombe et Périés étaient dépêchés pour faire réparer les routes les plus dégradées, tandis que Bion, Dautriche, Estadens et Mirande étaient chargés de l'inspection du service des postes.

(65) AN, 1A 11, Bulletin des lois, $\mathrm{n}^{\circ} 164$; Proclamation du Directoire exécutif du 21 frimaire an VI (11 décembre 1797) relatif à la réparation des routes.

(66) Selon Francis D'IVERNOIS, seules deux communes auraient répondu à l'appel patriotique, Colonges et Verneuil : Tableau historique et politique des pertes que la Révolution et la guerre ont causées au peuple français, 1799, t. 1, p. 93. 
croisements sur près de 70 kilomètres, il suffit d'établir une barrière à Valence à l'embranchement de la route avec celle qui mène au Mont-Blanc ${ }^{67}$.

La perception du droit de passe qui débute en juillet 1798 dans plusieurs circonscriptions, présente rapidement d'évidentes difficultés : non seulement le nombre des barrières est insuffisant, mais encore l'emplacement de certaines d'entre elles, décidé par le ministère, laisse aux usagers la possibilité de les contourner. Pour limiter les risques d'évasion fiscale, le Directoire confie aux départements en thermidor an VI (juillet 1798) le soin de fixer le nombre des barrières et de déterminer les emplacements les plus appropriés au vu des situations locales. C'est ainsi qu'à l'échelle départementale, on retrouve de fortes variations dans la répartition locale des points de perception. Quand l'affermage des barrières est décidé, leur localisation fait l'objet d'une procédure de révision de façon à s'ajuster au mieux aux usages de la circulation : des barrières sont ajoutées, d'autres déplacées. La circulaire du 18 brumaire an VII ( 8 novembre 1798) demande par ailleurs à chaque département d'établir un plan topographique afin de réaliser une carte des barrières pour l'ensemble du territoire français ${ }^{68}$. Il resterait toutefois à déterminer dans quelle mesure la localisation de ces barrières reprend l'emplacement des bureaux de perception où étaient exigés les droits féodaux.

La loi du 3 nivôse an VI (23 décembre 1797) fixe le tarif des droits à percevoir sur les routes ainsi que les modalités de leur perception ${ }^{69}$. La grande innovation du Directoire est d'avoir corrélé, sur le modèle anglais, la tarification des infrastructures routières avec la police du roulage, afin de proportionner le montant des droits aux dommages que les usagers font subir à la chaussée ${ }^{70}$ : les roues des voitures lourdement chargées, en

(67) $\mathrm{AN}, \mathrm{F}^{14} 10026$ : Rapport fait par Pierre Dedelay sur la résolution du 29 frimaire an VI concernant le droit d'entretien des routes (séance du 3 frimaire an VI).

(68) Des cartes ont été dessinées en l'an VII à l'occasion de l'affermage des barrières (AN, $\mathrm{F}^{14}$ 10165-10190). Certains départements n'y ont toutefois mentionné que la désignation des bureaux sans préciser le nombre des barrières en dépendant.

(69) AN, 1A/11, Bulletin des lois, $\mathrm{n}^{\circ} 171$.

(70) Dès les premières années de la Révolution, on trouve chez Chaumont de la Millière, intendant général des Ponts et Chaussées (AN, AD XIII 16. Mémoire sur le département des Ponts et Chaussées, 1790), Jean-Marie Roland ministre de 1'Intérieur (AN, F ${ }^{14} 10026$. Rapport de Roland de la Platière, 6 janvier 1793), et Mahuet, ancien régisseur général des messageries, la même volonté de coupler le péage et la réglementation du roulage : par le biais de la fiscalité, on pouvait inciter les voituriers à modifier la construction de leurs véhicules, et moduler le tarif de façon à pénaliser les voituriers qui ne respecteraient pas les prescriptions en matière de police du roulage. Afin de salarier des cantonniers (ou stationnaires) affectés à l'entretien des routes, Mahuet proposait, dès 1790, l'établissement d'un « droit de voirie » qu'il fixait à un denier par lieue et par quintal. En germinal an VIII, il défend l'idée d'un droit d'un centime par quintal et par lieue de poste sur la charge que chaque voiture est 
passant dans les interstices laissés entre les pavés, tendent à les écarter et à briser les angles ; sur les axes empierrés, les jantes entaillent la surface de cailloutis. Non seulement le tarif est dégressif pour les charrettes et chariots munis de jantes larges de façon à inciter les voituriers à en équiper leurs voitures, mais encore il pénalise les chargements lourds : il est calculé en fonction du nombre de chevaux composant l'attelage - plus ils sont nombreux, plus la cargaison doit être lourde - ou après une pesée des voitures sur des ponts à bascule ${ }^{71}$.

À la réglementation édictée par la monarchie au cours du XVIII siècle pour limiter le chargement des véhicules en restreignant la force de traction autorisée, le Directoire ajoute ainsi le levier fiscal pour obliger les rouliers à répartir leurs cargaisons sur plusieurs voitures. Le droit à acquitter correspond par ailleurs au trajet effectué sur une distance donnée ${ }^{72}$. La loi du 14 brumaire an VII (4 novembre 1798) prescrit que la perception de la taxe doit se faire, non pas pour le chemin parcouru mais pour l'espace à parcourir entre deux barrières (article IX). Le droit est dû de façon forfaitaire pour la distance comprise entre la première barrière franchie et la suivante, quel que soit le trajet réel effectué sur cette portion de route ${ }^{73}$.

(Suite de la note 70 page 24.)

autorisée à transporter ainsi que 15 centimes par lieue de poste et par roue pour les voitures suspendues ou non transportant des voyageurs devrait pourvoir au parfait rétablissement des routes : $\mathrm{AN}, \mathrm{F}^{14}$ 954/3. Observations sur le droit de passe et sur les moyens les plus convenables pour pourvoir à la réparation et à l'entretien des routes commerciales et pour prévenir par la suite leur dégradation, an VIII.

(71) Dès 1784, Chaumont de la Millière avait proposé au contrôleur général Calonne de vérifier le poids des voitures sans les décharger grâce à des ponts à bascule conçus par Mahuet. Il renouvela ses propositions en 1790 dans son Mémoire sur l'entretien des routes commerciales (AN, AD XI 560). Quelques expérimentations limitées avaient été tentées avant la Révolution. Avec l'instauration du droit de passe, des ponts à bascule permirent de peser les voitures des rouliers, et de surtaxer celles qui seraient surchargées. Ce n'est que la loi du 29 floréal an X (19 mai 1802) qui généralisa ces ponts à bascule, et les préposés aux opérations de pesage furent aussi chargés de vérifier la largeur des bandes des voitures employées au roulage et aux messageries. Le pesage des voitures constitue un problème car les matériels dont dispose le commerce sont peu pratiques et lents.

(72) AD Seine-Maritime, L 1074 : Tarif général de la taxe d'entretien sur les routes par cinq kilomètres. Sur cette base, le droit était ensuite majoré d'un centime par tronçon supplémentaire dont la distance était comprise entre $13,888 \mathrm{~m}$ et $1 \mathrm{~km}$ selon le mode de transport. Par exemple, il en coûtait 10 centimes pour une charrette à deux roues tirée par un cheval sur une distance de cinq kilomètres ; tous les $500 \mathrm{~m}$ effectués en sus, le montant de la taxe augmentait d'un centime.

(73) Cette disposition fit l'objet de vives critiques. J. Cordier pointait ainsi le caractère injuste de l'article 15 qui portait que « le tarif de la taxe doit être réglé ainsi : pour une distance entre deux barrières de 2500 à $7500 \mathrm{~m}$, la taxe sera perçue sur le pied de $5000 \mathrm{~m}$; de 7500 à $12500 \mathrm{~m}$, sur le pied de $10000 \mathrm{~m}$ et ainsi de suite. Il résultait de ces dispositions erronées que le voiturier payait quelquefois la même somme à avoir parcouru $2051 \mathrm{~m}$ ou $7499 \mathrm{~m}$, ou une somme double après un trajet de $7501 \mathrm{~m}$. Ainsi la taxe était tantôt la même pour une distance triple, et tantôt double pour deux distances presque identiques » (Essai sur la construction des routes, des canaux et la législation des Travaux publics, Lille 1823). 
Deux barrières doivent être distantes d'au moins $2,5 \mathrm{~km}$. De la même façon, l'octroi de navigation créé sous le Consulat va être calculé à l'avalage comme à la remontée en fonction de la distance qui sépare deux bureaux de perception « sans avoir égard au point de départ ni à celui de débarquement ${ }^{74}$. C'était une façon de ne pas multiplier les points de perception et de limiter les arrêts préjudiciables à la fluidité de la circulation. Adopter la distance comme métrique de l'espace des communications renvoie certes à un souci de continuité territoriale; ce mode de tarification pose toutefois problème dans la mesure où ne sont pas pris en considération la configuration de la route et le renfort éventuel de l'attelage pour des itinéraires accidentés.

Pour la « taxe de navigation » que M. Challan propose dès l'an VII pour financer l'entretien des voies d'eau et réintroduire ce faisant une parité fiscale entre les deux modes de transport, le cours de chaque fleuve devait être divisé en trois sections : l'embouchure où la navigation est tant maritime que fluviale ; puis jusqu'au lieu où la navigation commence à décroître ; enfin l'amont jusqu'au lieu où elle devient impossible. La partie intermédiaire devait servir de base à la tarification; la taxe serait respectivement doublée à l'aval et divisée par deux à l'amont. Pour les rivières affluentes, la quotité de la taxe serait déterminée par le lieu de confluence avec le fleuve ${ }^{75}$. L'utilité d'un cours d'eau étant décroissante, l'idée est de faire varier la quotité de la taxe en proportion. Une étude reste cependant à mener pour déterminer si ces prescriptions ont été retenues pour l'établissement du tarif de l'octroi de navigation, chaque bassin fluvial étant subdivisé en arrondissements numérotés par ordre croissant de l'amont à l'aval.

Cette indexation proportionnelle du prix du péage à la distance s'inscrit par ailleurs dans une conception concentrique de l'espace de circulation. En 1790, Mahuet a appuyé son projet de taxe sur une modélisation auréolaire, sous la forme d'aires juxtaposées et de circuits courts dans le cadre d'une économie décentralisée : «Les rayons du cercle de la France, considérés sous les rapports commerciaux \& dans une latitude moyenne, peuvent être calculés à 50 lieues $»^{76}$. À l'échelle de la Répu-

(74) AN, H4 3203. Arrêté contenant règlements pour la perception de l'octroi de navigation dans les neuf arrondissements du bassin de la Seine (19 messidor an XI).

(75) AN, H4 3130 : Rapport et projet de résolution présenté par M. Challan sur la navigation intérieure et fluviale (27 prairial an VII).

(76) AN, AD XI 560 : MAHUET, Mémoire sur l'entretien des routes commerciales du royaume présenté à l'Assemblée Nationale, 1790. 
blique, le physiocrate Fr.-René de Pommereul affirme quant à lui que « le mouvement des routes baisse en allant du centre vers la circonférence $[\ldots] \gg^{77}$.

À partir d'une réflexion qui croise à un principe auréolaire une logique nodale pour localiser un grand nombre de bureaux de perception, le réseau s'affirme comme une composante dynamique d'un système territorial. Il resterait cependant à déterminer si ces politiques tarifaires ont eu une incidence sur la géographie des transports marchands, et dans quelle mesure les usages de la circulation peuvent expliquer l'échec de la taxe d'entretien des routes et son abandon en 1806.

L'espace des transports tel qu'il se dessine à travers les débats et les mémoires de l'époque est donc très largement le fruit d'une construction abstraite, qui s'affirme précocement dans l'appareil idéologique et politique de la Révolution. La façon de penser cet espace relève très largement d'un projet normatif qui, tout en intégrant l'héritage de l'Ancien Régime et en composant avec les contraintes économiques du moment, participe à l'affirmation d'une nouvelle échelle territoriale. Il s'agit, dans un cadre juridique et administratif unifié, de promouvoir un système de communications qui doit très largement œuvrer à l'unité du territoire. Dans cet esprit, la politique d'aménagement suppose de repenser l'articulation des axes de circulation, à commencer par les rapports de complémentarité entre les deux modes de transport. La continuité du territoire est également postulée avec l'adoption d'un référent neutre, la distance, pour fixer le maximum des prix de transport comme pour calculer le coût des péages nouvellement établis. De la même façon, avec la péréquation des recettes de la taxe d'entretien ${ }^{78}$, la dimension spatiale de la Nation prime sur l'intérêt local d'une liaison routière. Ces choix politiques, qui renvoient à une idéologie du territoire, avaient clairement pour but de favoriser la

(77) François-René-Jean DE Pommereul, Observations sur le droit de passe proposé pour servir à la confection et l'entretien des chemins, janvier 1797.

(78) Defermont et Marragon, deux des principaux artisans de la taxe d'entretien des routes, avaient expressément réfuté l'idée smithienne d'un droit qui ne profite qu'à l'axe sur lequel il était exigé, pour lui préférer une taxe capable d'égaliser les niveaux d'équipement routier sur l'ensemble du territoire. Cette compensation nationale joua pleinement, permettant aux départements les plus enclavés (dans le Centre et le Midi) de bénéficier des profits dégagés par ceux qui étaient les plus fréquentés. Il va en être autrement pour l'octroi de navigation dont les «produits formeront des masses distinctes ; et l'emploi en sera fait limitativement sur chaque canal, fleuve et rivière sur lesquels la perception aura été faite » (Loi relative à l'établissement d'un droit de navigation intérieure, 30 floréal an X). La collecte et la gestion de ces fonds se feront à l'échelle départementale. $C f$. AN, $\mathrm{F}^{14} 428$ et suiv. 
construction d'un marché national et de stimuler les dynamiques économiques. Il n'en reste pas moins que cet espace isotrope et homogène que postulent les autorités, étalonné par la seule distance, fait largement abstraction des contraintes du relief comme de la géographie des marchés, la circulation marchande étant à l'époque encore essentiellement fondée sur des échanges locaux et inter-régionaux.

Anne CONCHON

Université Paris 1 - IDHE 17 , rue de la Sorbonne 75231 Paris Cedex 05 conchon@univ-paris1.fr 